\title{
Management of Non-Classic Congenital Adrenal Hyperplasia in Pregnant Woman - Non-Referral Center Experience- Case Report
}

\author{
Zoran Gluvic ${ }^{1}$, Milena Lackovic ${ }^{1}$, Vladimir Samardzic ${ }^{1}$, Bojan Mitrovic ${ }^{1}$, Violeta \\ Mladenovic ${ }^{2}$, Milan Obradovic ${ }^{3}$, Danimir Jevremovic ${ }^{4}$ and Esma $R$ Isenovic ${ }^{3 *}$ \\ ${ }^{1}$ Department of Endocrinology and Diabetes, Zemun Clinical Hospital, University of Belgrade, Serbia \\ ${ }^{2}$ Clinical Centre Kragujevac, Clinic for Endocrinology, University of Kragujevac, Serbia \\ ${ }^{3}$ Institute of Nuclear Sciences Vinca, University of Belgrade, Laboratory of Radiobiology and Molecular Genetics, Serbia \\ ${ }^{4}$ Faculty of Stomatology in Pancevo, University Business Academy, Serbia
}

*Corresponding author: Prof. Dr. Esma R. Isenovic, Institute of Nuclear Science Vinca, University of Belgrade, Laboratory of Radiobiology and Molecular Genetics, P.O. Box 522, 11000 Belgrade, Serbia, Tel/ Fax: +38111-3408147

\begin{abstract}
Congenital adrenal hyperplasia $(\mathrm{CAH})$ is a rare autosomal recessive disorder with mutations in genes involved in cortisol and aldosterone production. Based on overall 21-OHase activity, $\mathrm{CAH}$ is divided into classic $(\mathrm{C}-\mathrm{CAH})$ and non-classic (NC-CAH). Females who suffered from NC-CAH have had increased infertility rates and higher miscarriage susceptibility. The treatment of $\mathrm{CAH}$ in pregnancy is still debatable.

We present 22-years-old pregnant female (seventh week of gestation), who is currently under dexamethasone (DEX) since almost seven years for NC-CAH. At presentation, she is normotensive, non-obese, with no signs of hirsutism and Cushing syndrome. Seven days after the first visit, an endocrinologist makes informative talk with the patient and her mother about NC-CAH, pregnancy, and drugsassociated risks. Current Clinical Practice Guideline for $\mathrm{CAH}$ treatment suggests the use of protocols approved by Institutional Review Boards at Centers experienced in CAH treatment.

In women with $\mathrm{CAH}$ who are planning a pregnancy, a close relationship between endocrinologist, reproductive gynaecologist and molecular biologist is of great interest. Prenatal management with DEX is advised in particular circumstances. In remaining, the switch from DEX to other glucocorticoids that do not penetrate placenta is advised.
\end{abstract}

\section{Keywords}

Congenital adrenal hyperplasia, Miscarriage, Pregnancy, Glucocorticoids, Dexamethasone, Hydrocortisone

\section{Introduction}

Congenital adrenal hyperplasia (CAH) represents a group of inherited diseases, caused by autosomal recessive mutation of genes involved in adrenal cortex hormone synthesis: glucocorticoids (GC) and mineralocorticoids (MC) [1,2]. The predominant mutations in CAH individuals are located in gene encoding 210H-ase (CYP21A2) [3]. CAH can be divided into classic $\mathrm{CAH}$ (C-CAH) and non-classic CAH type (NC-CAH) with $<5 \%$ and $20-50 \%$ preserved $210 \mathrm{OH}$-ase activity, respectively $[2,4]$. In comparison to C-CAH, NCCAH is more common (about 1:1000 to 1:2000 births), especially in certain ethnic groups, such as Ashkenazy Jews (1 in 27 to 1 in 30 cases) [5]. Approximately 70\% of patients with NC-CAH have a point mutation of Val281Leu at exon 7, and $27-76 \%$ of patients have a severe mutation $[6,7]$. If both partners are the carriers of the severe mutation, the child of them could inherit C-CAH [6].

Clinical presentation of NC-CAH in adult women is very diverse, from asymptomatic to one presented as hirsutism, menstrual disorders, acne, infertility and rarely primary amenorrhea and virilization [8]. In males, NC-CAH is usually asymptomatic but can be the cause of impaired fertility [8]. The diagnostic procedure for NC-CAH involves measurement of 17-OHP, ACTH stimulation test, and genetic testing for CYP21A2

Citation: Gluvic Z, Lackovic M, Samardzic V, Mitrovic B, Mladenovic V, et al. (2019) Management of Non-Classic Congenital Adrenal Hyperplasia in Pregnant Woman - Non-Referral Center ExperienceCase Report. Clin Med Rev Case Rep 6:257. doi.org/10.23937/2378-3656/1410257

Accepted: February 20, 2019: Published: February 22, 2019

Copyright: (c) 2019 Gluvic Z, et al. This is an open-access article distributed under the terms of the Creative Commons Attribution License, which permits unrestricted use, distribution, and reproduction in any medium, provided the original author and source are credited. 
mutation $[7,9]$. Genetic testing is preferably performed in symptomatic NC-CAH. Most of screening programs do not detect individuals with NC-CAH because of the majority of individuals with NC-CAH experience normal growth, puberty, and reproduction [10].

Treatment of NC-CAH is recommended in symptomatic individuals with the aim to reduce clinically apparent hyperandrogenism. Hydrocortisone (HC) in children and glucocorticoids (GCs) other than HC (prednisone, prednisolone, and DEX) are preferable treatment options $[7,11]$.

We present a case of a woman with NC-CAH and unexpected pregnancy, who was already under treatment with DEX. Its use in pregnancy is still debatable, because of mothers' and fetus' short- and long term risks.

\section{Discussion}

Biochemistry analyses were performed by DxC 800 and DXI-600 Beckman Coulter devices. Reference intervals for biochemical parameters: glycaemia, sodium and potassium were $3.5-5.5 \mathrm{mmol} / \mathrm{L}, 138-149 \mathrm{mmol} / \mathrm{L}$, and $4.0-5.0 \mathrm{mmol} / \mathrm{L}$, respectively. Reference intervals for measured hormones: testosterone, adrenocorticotropic hormone (ACTH), 17-hydroxyprogesterone (17$\mathrm{OHP}$ ) and thyroid stimulating hormone (TSH) were 0.5$2.6 \mathrm{nmol} / \mathrm{L}, 10-80 \mathrm{pg} / \mathrm{ml}$, follicular phase and ovulation $<1.3$ and $<4.5 \mathrm{mcg} / \mathrm{L}$, as well as $0.35-5.5 \mathrm{mlU} / \mathrm{ml} \mathrm{re}$ spectively. Noninvasive prenatal diagnostics was done by Tranquility test (Genoma Swiss Biotechnology, Geneva, Switzerland). It is capable of recognizing the most frequent numeric aberrations and fetal gender in early pregnancy (10 weeks of gestation) by analyzing mother's blood.

Written informed consent was obtained from the patient, and the Ethical Committee of Zemun Clinical Hospital approved the study.

We present 22-years-old pregnant female (seventh week of gestation), who is currently under DEX treatment began since almost seven years for NC-CAH $(0.25$ $\mathrm{mg}$ on working days, $0.5 \mathrm{mg}$ on weekdays), diagnosed during the evaluation of secondary amenorrhea. She did not carry out any advised genetic counselling. At presentation, she is normotensive, slim (Body Mass Index $19.5 \mathrm{~kg} / \mathrm{m}^{2}$ ), with no hirsutism (Ferriman-Lorenz-Gallway scale of hirsutism $<16$ ) and clinical signs of Cushing syndrome. Previous and current results are shown in Table 1. Seven days after the first visit, an endocrinologist makes informative talk with the patient and her mother about NC-CAH and pregnancy, drugs and associated risks. DEX was switched to HC $(20 \mathrm{mg} /$ bid). Prenatal genetic testing was done (tenth week of gestation) and revealed the female gender of the fetus with no gross quantitative chromosomal abnormalities. HC treatment was succeeded, and regular follow-up of mother and fetus was continued, with no visible changes in fetal morphology.

Infertility affects about $13 \%$ of women with NCCAH but can be improved with HC treatment $[6,12]$. Women with NC-CAH who have a desire to be pregnant must bear in mind the risk of C-CAH in infant [13]. In the study of Bidet, et al. $1.5 \%$ of the infants were born with C-CAH from the population of mothers with NC$\mathrm{CAH}$ [6]. Another study reported a higher risk of C-CAH in newborns $(2.5 \%)$, and that about $15 \%$ of offspring would have NC-CAH [14]. Predicted risk of having a child with C-CAH of NC-CAH parents is 1:240. According to the fact that the vast majority of NC-CAH parents are heterozygous compound carriers of severe and mild mutations, further predicted incidence is about 1:360 $[7,15]$.

Pregnancies with NC-CAH are thought as high-risk, because of higher rates of miscarriages, predominantly in those who are not covered with GCs (26.3 vs. $6.5 \%)$ [6]. According to the report that the administration of $\mathrm{HC}$ before and during the pregnancy period reduced miscarriage rates [6], the women with $\mathrm{CAH}$ who

Table 1: The results' cross-section of biochemical and hormonal analyses and administered drugs.

\begin{tabular}{|c|c|c|c|}
\hline Analysis & 2016 & 2017 & 2018 (7WG) \\
\hline Potassium $\left(\mathrm{K}^{+}\right)[\mathrm{mmol} / \mathrm{l}]$ & 4.5 & 4.6 & 3.9 \\
\hline Sodium $\left(\mathrm{Na}^{+}\right)[\mathrm{mmol} / \mathrm{l}]$ & $136(\downarrow)$ & 137 & $135(\downarrow)$ \\
\hline Glycaemia (Gly) [mmol/l] & & 4.9 & 4.5 \\
\hline Testosterone [nmol/L] & 2.1 & 1.2 & 2.48 \\
\hline 17-OHP [mcg/L) & $15.7(\uparrow)$ & $5.0(\uparrow)$ & $12.7(\uparrow)$ \\
\hline ACTH [pg/ml] & & & 11.9 \\
\hline TSH [mlU/ml] & & & 0.85 \\
\hline Current & DEX: & DEX: & Switch to $\mathrm{HC}$ \\
\hline DEX dose & $0.25 \mathrm{mcg}$ & $\begin{array}{l}0.25 \mathrm{mcg} / 5 \text { days weekly, } \\
0.5 \mathrm{mcg} / 2 \text { days weekly }\end{array}$ & \\
\hline Recommended & DEX: & DEX: & $\mathrm{HC}: 15+05+0 \mathrm{mg}$ \\
\hline DEX/HC dose & $\begin{array}{l}0.25 \mathrm{mcg} / 5 \text { days weekly, } \\
0.5 \mathrm{mcg} / 2 \text { days weekly }\end{array}$ & $\begin{array}{l}0.25 \mathrm{mcg} / 5 \text { days weekly, } \\
0.5 \mathrm{mcg} / 2 \text { days weekly }\end{array}$ & \\
\hline
\end{tabular}

WG: Week of Gestation; 17-OHP: 17-hydroxyprogesterone; ACTH: Adrenocorticotropic Hormone; TSH: Thyroid Stimulating Hormone; DEX: Dexamethasone; HC: Hydrocortisone; $\downarrow$ : decreased; $\uparrow$ : increased. 
were under treatment with GCs before pregnancy will continue the treatment during pregnancy, but with GCs that do not cross the placenta, such as HC, prednisone, and prednisolone administered in the doses used in secondary adrenocortical failure [9]. So, the recommended daily dose of those treated with $\mathrm{HC}$ was 20-25 mg from the beginning of pregnancy [6]. On the contrary, the women with NC-CAH who were not managedwith GCs before the pregnancy are not candidates for GCs introduction during pregnancy [16].

The reasons for DEX use in pregnancy are the prenatal treatment of female fetus that is at risk of $\mathrm{C}-\mathrm{CAH}$-associated virilization, the need for fetus reconstructive surgery and anxiety control of parents who might have a child with ambiguous genitalia $[11,15,16]$. According to all, DEX must be introduced just after pregnancy confirmation (at six weeks), because fetal genital virilization begins six to seven weeks after conception [16]. The recommended DEX use is $20 \mathrm{mcg} / \mathrm{kg}$ of maternal body weight divided into three doses. DEX is not inactivated by 11-betahydroxysteroid dehydrogenase type II, so it passes the placenta and suppresses fetal ACTH secretion [17]. If the prenatal diagnostics are suggestive to a male fetus, DEX treatment is discontinued [17].

Regarding delayed results of genetic testing in individuals who underwent chorionic villous biopsies (performed at ten to twelve weeks), all pregnancies at risk for fetal C-CAH are treated even though the only 1 of 4 is affected, and 1 in 8 affected fetuses is female $[15,18]$. As the prenatal treatment of $\mathrm{CAH}$ is still experimental, the best approach is to introduce DEX treatment in specialized and referral centres with relevant Ethical Committee, after the mother signed written informed consent [9]. Except in already mentioned reasons, current Endocrine Society Clinical Practice Guideline suggests avoiding of prenatal DEX exposure of mother and fetus and consequent potential harmful effects of DEX use, that are not thoroughly examined [11].

DEX is classified as $B$ category drug regarding safety in pregnancy, which means that safety in pregnancy, is not established. So its prescription for prenatal treatment of CAH is off-label in USA and EU [11]. Both fetus and mother are exposed to the risk of DEX use in pregnancy. The fetus risk associated with GCs use in early pregnancy includes orofacial clefts, reduction in short-term, non-verbal and verbal working memory $[15,19,20]$. In mothers, GCs-induced hypercortisolism could be the consequence of GCs treatment [21]. Additionally, significance of collaboration between gynaecologists and reproductive endocrinologist must be stressed in regards to DEX use in assisted reproductive technologies. According to that, the reduction of prenatal use of DEX will reduce long-term cognitive disorders in treated healthy female fetuses [4].

\section{Conclusions}

We present a case of unexpectedly pregnant young women, who was already under the DEX treatment because of NC-CAH-induced secondary amenorrhea seven years. Taking into account that she was not sure about pregnancy continuation, her mental condition, the refuse of partner to undergo genetic test regarding $\mathrm{CAH}$ detection, as well as the fact that DEX was already changed into $\mathrm{HC}$, the multidisciplinary team approved further $\mathrm{HC}$ use and thorough follow-up.

In conclusion, switching of DEX to other GCs that do not penetrate placenta is of great importance in early pregnancy, before the virilization of fetuses begins. The use of modern prenatal genetic testing can additionally help the clinician in deciding on further use of GCs during pregnancy. The use of DEX is appropriate in strongly recommended cases. It is suggested that the introduction and surveillance of DEX treatment in pregnant women with NC-CAH is reserved for referral and experienced centres.

\section{Acknowledgements}

This work is supported by the grants No. 173033 (to E.R.I.) from the Ministry of Science, Republic of Serbia.

\section{Disclosure of Interest}

The authors report no conflict of interest.

\section{References}

1. El-Maouche D, Arlt W, Merke DP (2017) Congenital adrenal hyperplasia. Lancet 11: 2194-2210.

2. Gluvic Z, Samardzic V, Zaric B (2017) Terminal chronic kidney disease with arterial hypotension in a patient with classic congenital adrenal hyperplasia. Medical Investigations (Serbian) 15: 29-33.

3. Joehrer K, Geley S, Strasser-Wozak EM, Azziz R, Wollmann HA, et al. (1997) CYP11B1 mutations causing non-classic adrenal hyperplasia due to 11 beta-hydroxylase deficiency. Human Molecular Genetics 6: 1829-1834.

4. Miller A, Malek R (2018) Management of pregnancy in a patient with nonclassical congenital adrenal hyperplasia. AACE Clinical Care Reports 4: e163.

5. Speiser PW, Dupont J, Zhu D, Serrat J, Buegeleisen M, et al. (1992) Disease expression and molecular genotype in congenital adrenal hyperplasia due to 21-hydroxylase deficiency. J Clin Invest 90: 584-595.

6. Bidet $M$, Bellanne-Chantelot $C$, Galand-Portier MB, Golmard JL, Tardy V, et al. (2010) Fertility in women with nonclassical congenital adrenal hyperplasia due to 21-hydroxylase deficiency. J Clin Endocrinol Metab 95: $1182-1190$

7. Trapp CM, Oberfield SE (2012) Recommendations for treatment of nonclassic congenital adrenal hyperplasia (NCCAH): An update. Steroids 77: 342-346.

8. Baron JJ, Baron J (1993) Differential diagnosis of hirsutism in girls between 15-19 years old. Ginekologia polska 64: 267-269.

9. Ambroziak U, Bednarczuk T, Ginalska-Malinowska M, Małunowicz EM, Grzechocińska B, et al. (2010) Congenital 
adrenal hyperplasia due to 21-hydroxylase deficiency management in adults. Endokrynol Pol 61: 142-155.

10. White PC (2009) Neonatal screening for congenital adrenal hyperplasia. Nat Rev Endocrinol 5: 490-498.

11. Speiser PW, Azziz R, Baskin LS, Ghizzoni L, Hensle TW, et al. (2010) Congenital adrenal hyperplasia due to steroid 21-hydroxylase deficiency: An Endocrine Society clinical practice guideline. J Clin Endocrinol Metab 95: 4133-4160.

12. Moran C, Azziz R, Carmina E, Dewailly D, Fruzzetti F, et al. (2000) 21-Hydroxylase-deficient nonclassic adrenal hyperplasia is a progressive disorder: a multicenter study. Am J Obstet Gynecol 183: 1468-1474.

13. Cuhaci N, Aydin C, Yesilyurt A, Ferda Alpaslan Pınarli, Reyhan Ersoy, et al. (2015) Nonclassical Congenital Adrenal Hyperplasia and Pregnancy. Case reports in endocrinology 2015: 296924.

14. Moran C, Azziz R, Weintrob N, Witchel SF, Rohmer $\mathrm{V}$, et al. (2006) Reproductive outcome of women with 21-hydroxylase-deficient nonclassic adrenal hyperplasia. J Clin Endocrinol Metab 91: 3451-3456.

15. Miller WL, Witchel SF (2013) Prenatal treatment of congenital adrenal hyperplasia: Risks outweigh benefits. American Journal of Obstetrics and Gynecology 208: 354-359.
16. LWPES/ESPE CAH Working group (2002) Consensus statement on 21-hydroxylase deficiency from the Lawson Wilkins Pediatric Endocrine Society and the European Society for Paediatric Endocrinology. The Journal of Clinical Endocrinology and Metabolism 87: 4048-4053.

17. Lekarev O, New MI (2011) Adrenal disease in pregnancy. Best Pract Res Clin Endocrinol Metab 25: 959-973.

18. Witchel SF (2013) Non-classic congenital adrenal hyperplasia. Steroids 78: 747-750.

19. Hirvikoski T, Nordenstrom A, Lindholm T, Lindblad F, Ritzén EM, et al. (2007) Cognitive functions in children at risk for congenital adrenal hyperplasia treated prenatally with dexamethasone. J Clin Endocrinol Metab 92: 542-548.

20. Wallensteen L, Zimmermann M, Thomsen Sandberg M, Anton Gezelius, Anna Nordenström, et al. (2016) Sex-Dimorphic Effects of Prenatal Treatment With Dexamethasone. The Journal of Clinical Endocrinology and Metabolism 101: 3838-3846.

21. New MI, Carlson A, Obeid J, Marshall I, Cabrera MS, et al. (2001) Prenatal diagnosis for congenital adrenal hyperplasia in 532 pregnancies. J Clin Endocrinol Metab 86: 5651-5657. 